\title{
Clinical Risk Factors Predisposing to Vascular Cognitive Decline in Atherosclerotic Patients
}

Dempsey RJ, Wilbrand SM, Wesley U, Varghese T, Kalluri H and Hermann B Department of Neurosurgery, University of Wisconsin, Madison WI, USA

BACKGROUND: We have previously shown that in patients with significant large vessel atherosclerosis, the degree of vascular cognitive decline could be predicted by the instability of that atherosclerotic plaque, as measured by strain in the pulsating carotid artery plaque. We here study the role of family history of TIA and clinical risk factors in contributing to the relationship between vascular plaque strain and cognitive function.

METHODOLOGY: We studied 89 patients with significant (>60\%) atherosclerosis, 45 symptomatic and 44 asymptomatic. All were studied for vascular cognitive decline (NINDS battery) and vascular wall strain by ultrasound (Figure 1), as well as their major clinical risk factors and serum levels of inflammatory markers. Plaque strain values were log transformed for analysis by non-parametric method with significance set at $\mathrm{p}<0.05$. Due to most patients having one or more risk factors, we used risk factor(s) as inclusion criteria without any exclusion criteria to understand their contribution to the disease process. For biomarker identification, blood samples were collected from consented control subjects, and patients. We examined the differential adipokine levels in blood/plasma using a targeted proteome profiler adipokine antibody array.

RESULTS: A statistically significant relationship enhancing the plaque instability $(p=0.019)$ and cognitive decline in Digit Span function $(p=0.02)$ was seen with positive family history (Figure 2). This relationship is enhanced for digit span function by hyperlipidemia $(p=0.009)$, hypertension $(p=0.019)$ or carotid bruit $(p=0.005)$ which also works in a synergistic fashion $(p=0.002)$ (Figure 3A). However, under similar conditions, axial strain is enhanced by Hypertension and Carotid Bruit but not by Hyperlipidemia (Figure 3B). In a subset of 40 patients, proteomic antibody array showed significant increases in blood/plasma levels of the inflammatory markers IL1B $(p=<0.01)$, fibrinogen $(p=<0.05)$, and TIMP1 $(p=<0.05)$ were present in the symptomatic as compared to asymptomatic or control patients (Figure 4).

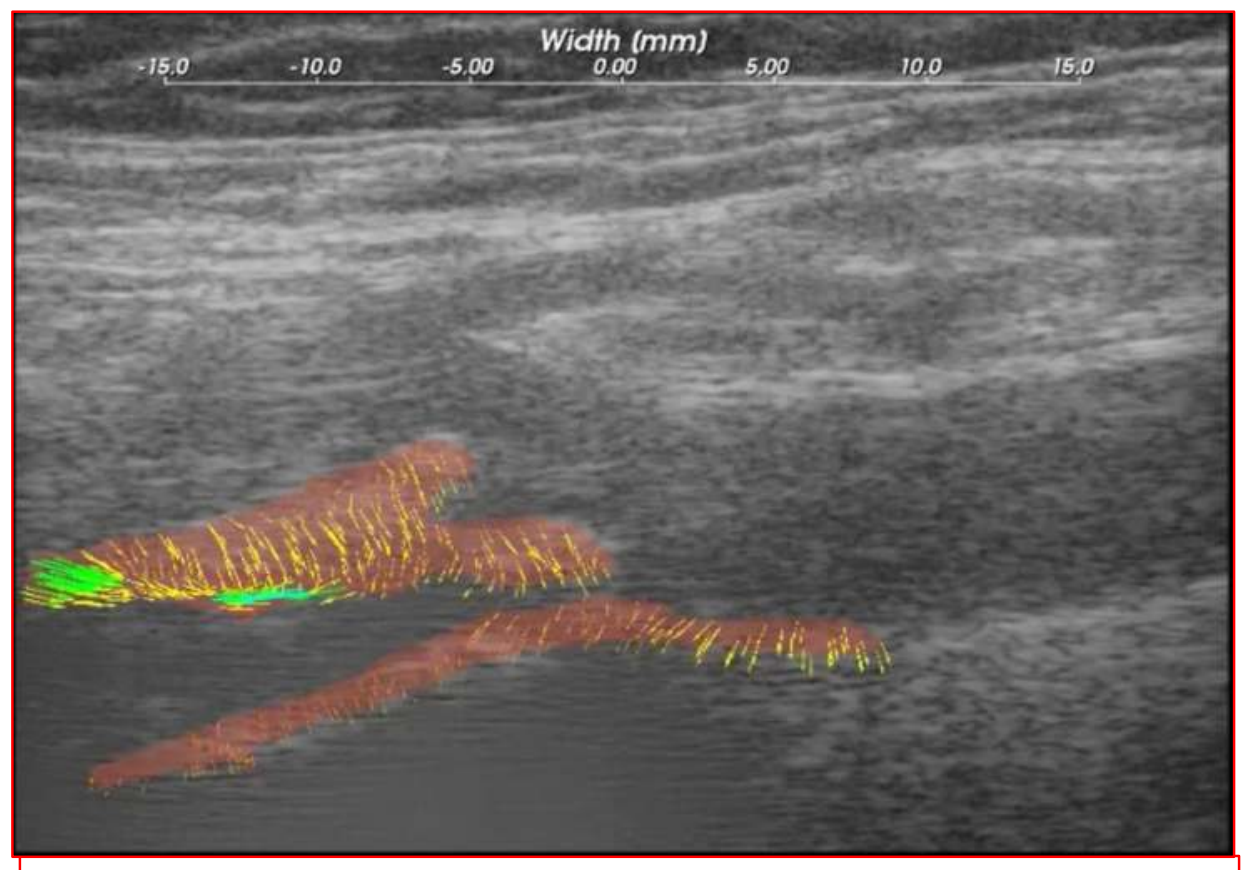

Figure 1. Ultra sound image of vessel wall with atherosclerotic plaque showing quantifiable strain lines within the pulsating plaque

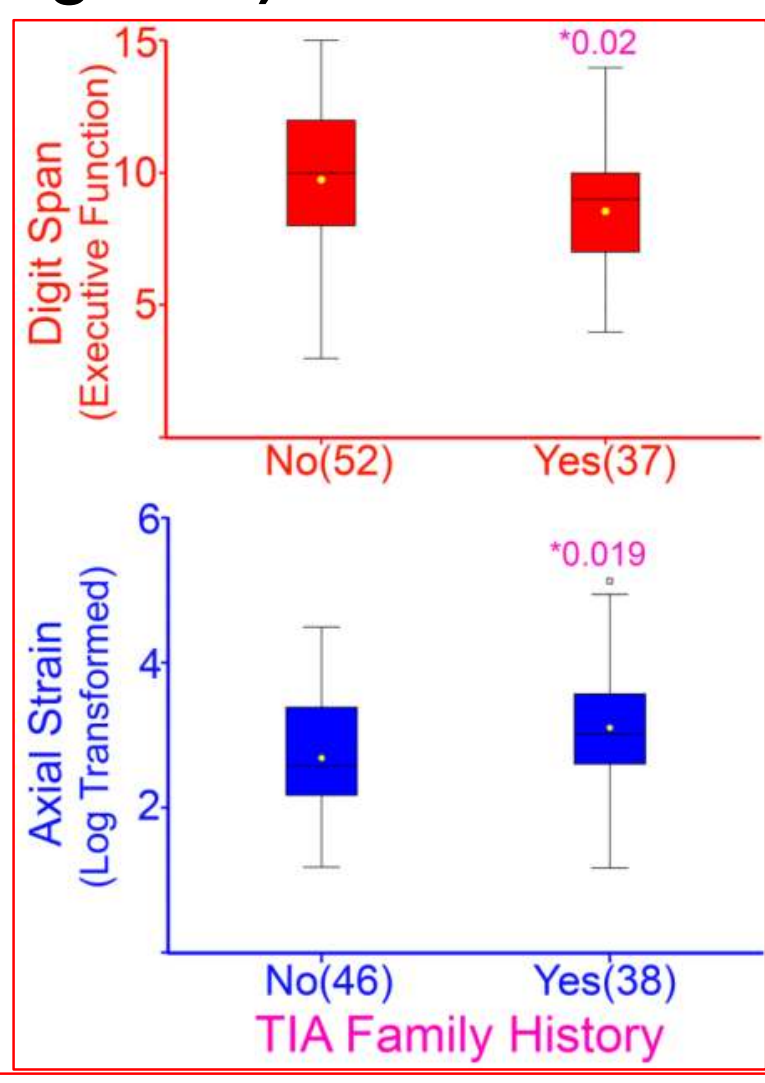

Figure 2. Effect of Family History on Digit Span and Axial Strain

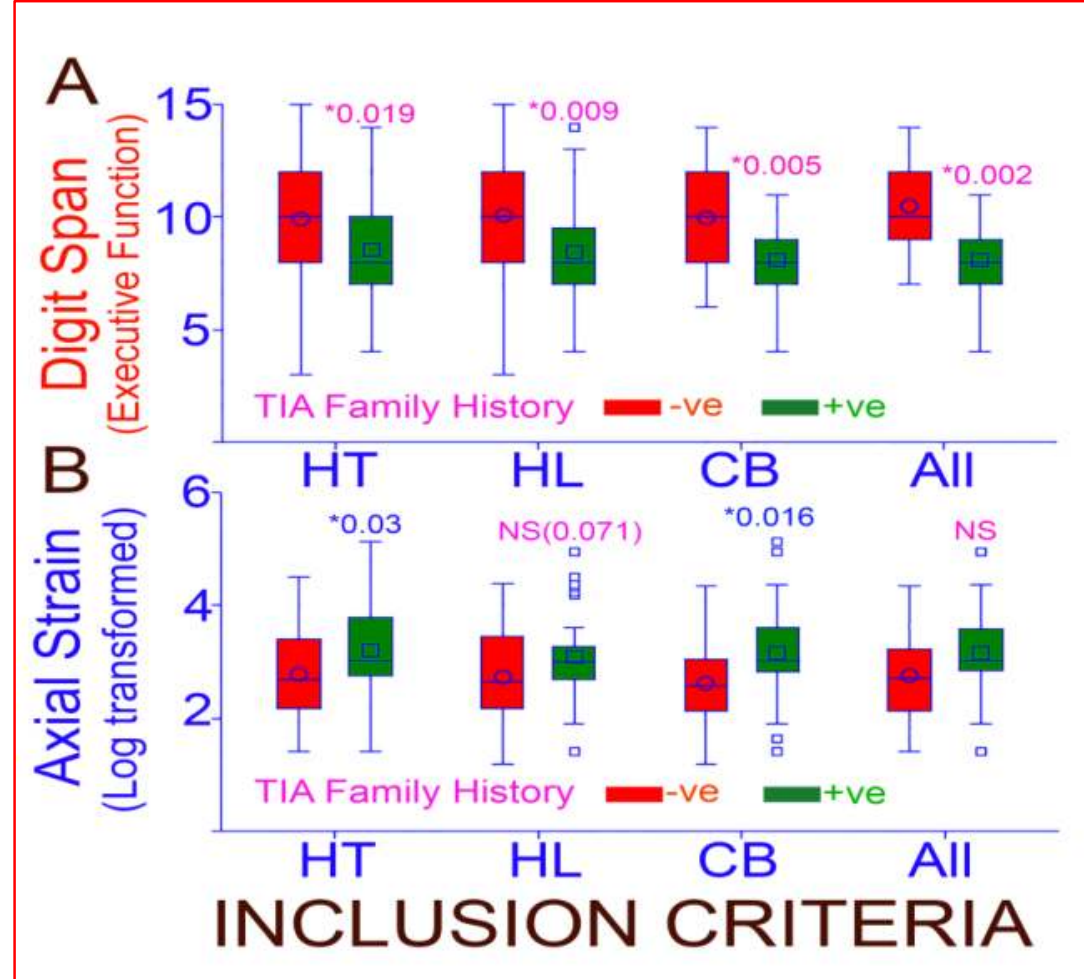

Figure 3. Effect of Risk Factors on Axial Strain \& Digit Span Function. HT: Hypertension HL: Hyperlipidemia CB: Carotid Bruit All: $\mathrm{HT}+\mathrm{HL}+\mathrm{CB}$

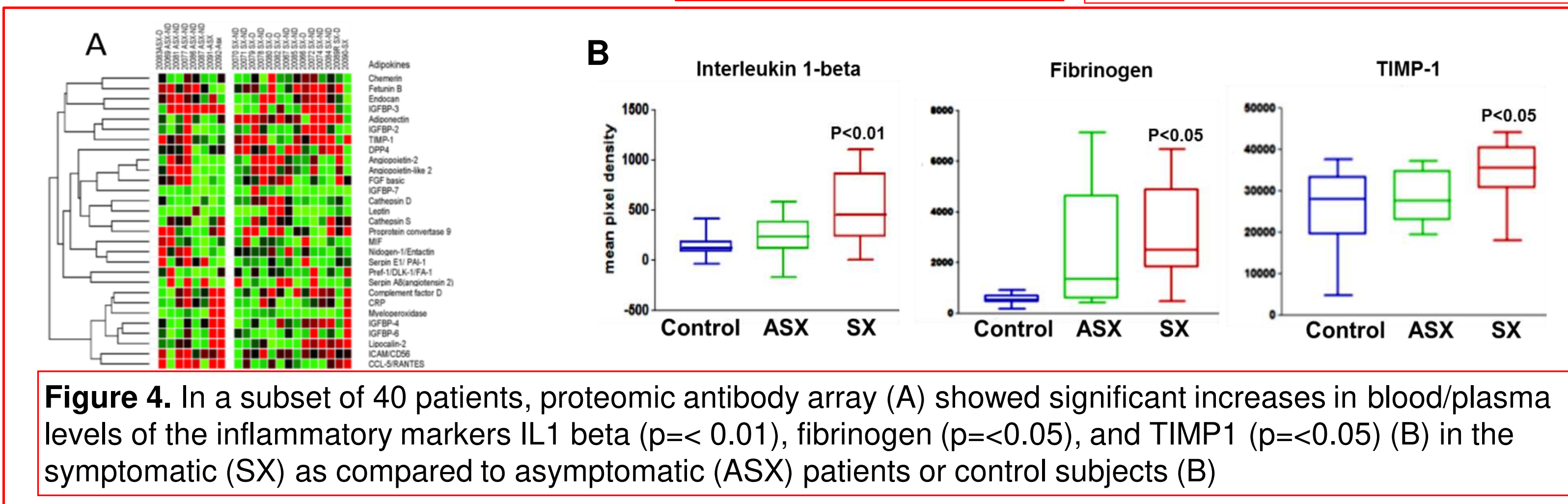

SUMMARY/CONCLUSIONS: These markers suggest a significant process of pro-inflammatory cytokines affecting adhesion molecules, viscosity, and tissue remodeling may play a systemic role in vascular cognitive decline for which instability of the carotid plaque may be a mechanical biomarker. This relationship is enhanced by the genetic and biochemical factors which determine family history, hyperlipidemia, and hypertension which could be modifiable factors in the process of vascular cognitive decline. Circulating proteomic signatures may serve as blood biomarkers of stroke risk and symptomology.

Acknowledgements: National Institute of Health; American Heart Association; Department of Neurosurgery; School of Medicine and Public Health, University of Wisconsin Madison, WI, USA 